\title{
Advice Not Taken
}

\section{Linda F. Bisson, Mary Lou de Leon Siantz, and Laura Grindstaff}

\begin{abstract}
Advice on how to build a more-inclusive academic community is emerging; however, this chapter suggests that such advice warrants "a grain of salt" depending on two circumstances: (1) the organizational culture needing to be "fixed," and (2) the existence of extra-organizational factors that may shape how transformation can proceed. First, the existing organizational culture affects the processes needed to achieve a more-inclusive community, and defines what "more inclusive" will look like. Programs shown to be effective at one institution might not be effective at another. External factors may also affect local culture. For example, a long-standing ban on affirmative action programs and quota systems at the University of California meant that, even though other institutions found them to be effective, replicating those programs was not an option. The second concern derives from the nature of change needed. Barriers to inclusion are deeply rooted in historical traditions, ideologies, and social practices outside of any single organization, and often these barriers are applied unconsciously. This means genuine cultural transformation will occur only if the organizational community as a whole is committed to that change.
\end{abstract}

Keywords Organizational culture $\cdot$ Inclusion $\cdot$ Diversity commitments $\cdot$ Cultural change $\cdot$ Accountability $\cdot$ Interventions $\cdot$ Transparency $\cdot$ Community learning

\section{F. Bisson ( $\varangle)$}

Department of Viticulture and Enology, University of California, Davis, Davis, CA 95616, USA e-mail: lfbisson@ucdavis.edu

M. L. de Leon Siantz

Betty Irene Moore School of Nursing, UC Davis Health System, University of California, Davis, 2315 Stockton Blvd., Sacramento, CA 95817, USA

e-mail: deleonsiantz@ucdavis.edu

L. Grindstaff

Department of Sociology, University of California, Davis, Davis, CA 95616, USA

e-mail: lagrindstaff@ucdavis.edu 


\section{Understanding the Nature of the Problem}

Understanding the nature of systemic bias in today's society is a critical first step in designing an effective strategy to create a more-inclusive community. The link between diversity, equity, innovation, and success has been clearly established (Davidson \& Goldberg, 2010). As a consequence, many organizations and institutions are committed to creating a more-inclusive community for themselves. Over 500 CEOs have signed a pledge for action on diversity and inclusion, having recognized the clear benefits to competitiveness (CEO Action for Diversity and Inclusion, n.d.). However, much of the work in organizational change focuses on modifying an internal culture-one that has long been established, vetted, and controlled by the organization (Quinn \& Cameron, 1988). Creating a more inclusive and equitable environment also requires that we address the impact and influence on local culture of broader, socio-cultural sources of inequality.

\subsection{Commitment of the Leadership is Necessary but Not Sufficient}

Guidelines for institutional transformation advise forming a powerful coalition early on that can lead the effort with enough authority to effect change (Kotter, 1995; see also Kalev et al., 2006). From the beginning, our ADVANCE leadership team headed this advice. We confronted a very important question: Who at our institution is powerful enough to change the very nature of society? The top academic leadership of an organization obviously plays a critical role in establishing a universal commitment to change and providing resources for implementing and sustaining it. However, the effort cannot stop there but must extend beyond the leader to include the community itself. By "community," we mean primarily but not exclusively the faculty, students, and staff in the STEM disciplines on our campus. A second central feature of our program, therefore, was to engage the community in learning about the nature and manifestations of bias and discrimination, both broadly and locally, as a way to vet the vision for the ADVANCE initiative. Shared knowledge can engender consensual cultural change at different levels of the institution.

Institutional transformation needs to be collective and communal, not top-down and imposed. In essence, effective change must be "led from the middle," meaning that it requires the support and commitment of the community at large in partnership with those connected to resources. In our case, assembling a powerful coalition of thoughtful leaders and engagers was critical to having difficult discussions to bring the academic community together around questions of culture. Analyses of why ADVANCE programs succeed or fail reinforce this approach. People will chase financial incentives and may adopt behaviors accordingly, but when the financial incentives end, the commitment to the vision may also end. Thus, there may be little correlation between initial investment and sustainable institutional transformation; 
missing is the ongoing communication needed to engage and maintain the partnerships that embrace diversity, equity, and inclusion (DEI) over time (Furst-Holloway et al., 2018).

\subsection{Ignoring Naysayers}

As we began our ADVANCE program at UC Davis, we were advised by those experienced with ADVANCE programming elsewhere to "ignore naysayers." After all, naysayers believed science should be independent of DEI concerns because such concerns could compromise excellence and innovation. We were warned about colleagues who simply "did not get it" and told we should exclude them from discussion. Dissenting voices were viewed as obstacles to achieving our goals. Yet if our goals encompassed "inclusivity," this advice was contradictory.

Although the voices of naysayers should not take up space disproportionate to their presence, we believe these voices should be embraced, for three important reasons. First, if the desired outcome is to be broadly inclusive, it can never be achieved by excluding dissenting views. Second, the perspectives gained from listening to both supporters and detractors provide the assessment data to help frame the action plan for changing the local culture. In other words, cultural transformation must be informed by assessing the nature of dissent. If engaged in the process, detractors help define obstacles to moving forward and, conversely, solutions for eliminating them. It is therefore important to foster a climate that welcomes constructive criticism, acknowledges that criticism exists, and is willing to address it appropriately. Finally, naysayers yield vital information on the spectrum of attitudes in the existing organizational culture and the extent to which historical barriers to inclusion are replicated within it. Assessing the nature and impact of barriers to inclusion first identifies the type of learning needed for institutional transformation and then helps guide optimal processes for such learning to occur.

\subsection{Develop a Common Foundation of Empathy and Commitment to Change, Not a Cadre of Allies}

Discussions of bias and discrimination in the academy and other institutions focus on the impact to women, men of color, and others from traditionally excluded or marginalized groups (identity categories). Often those in the majority are incorrectly seen as the sole cause of bias, as they disproportionately benefit from it. However, systemic biases emerge and get transmitted through individual attitudes, social interactions, cultural practices, and institutional structures that persist over time and affect everyone, albeit not in the same ways. Ideally, all community members should be viewed as stakeholders, regardless of identity. Consider the use of the term 'ally.' It 
is typically intended to indicate someone belonging to a privileged identity category who serves as a supportive 'bridge' to those from marginalized identity categories. All community members should be viewed as stakeholders regardless of identity. Treating everyone as stakeholders despite being differently situated with regard to systemic bias creates a culture of "we" and facilitates the types of difficult discussions about race, gender, class and other axes of identity and difference that need to occur.

As we learned more about the nature of barriers to inclusion, it became clear that no social identity, including majority ones, was free from stereotyping, exclusion, or implicit bias. At one roundtable, for example, a participant used the term "jerks" to describe those who opposed DEI efforts. Another participant suggested that we would not make progress if we persisted in "white male bashing." The first individual did not think she was "identity bashing," yet her words were interpreted that way. We also learned that some participants objected to the seemingly positive term "ally," because to them it meant one group siding with another; it reinforced an us-versus-them and sent the message that there was only one right way to do things. Putting members of any social identity category on the defensive is, unfortunately, a sure way to shut down meaningful discussion of inclusion. The brain has been described (admittedly simplistically) as having two components - the cognitive brain and the emotional brain, which are interconnected (Gray, 1990). Discussions of emotionally-charged topics like bias can trigger a strong emotional response. It is important, therefore, to maintain discussions in the cognitive sphere and to avoid triggers of noncognitive reactions.

As a consequence, we decided to include well-known white male stereotypes, such as "jerk," in discussions of the ways in which stereotyping creates barriers to inclusion. We were aware of and concerned about the potential to minimize the significantly greater negative impact of bias and discrimination on women, men of color, and other marginalized. However, we experienced the opposite-including stereotypes of white men enabled a more dynamic and productive discussion of the impact of stereotyping for all participants. Participants also expressed more intense interest in learning about other factors, aside from stereotyping, that create barriers to inclusion, such as implicit bias and role of social location in generating privilege/disadvantage. By recognizing the universal existence of negative stereotypes, we were able to build a common foundation of community empathy in these discussions, a necessary prerequisite to building a more equitable community.

None of this is to suggest that all categories of people are equally responsible for, or similarly affected by, the differential access to power, status, and resources generated by deeply-engrained social inequalities. Black people in the U. S. did not create nor do they benefit from anti-black racism, for example, whereas white people (often along with other non-black groups) did create and do benefit from it, albeit not in the same ways or to the same degree across all historical periods or all social contexts. Rather, we stress that some of the processes underlying and contributing to racism (as well as sexism, homophobia, ableism, etc.) — such as stereotyping and implicit bias-have more general application and this fact can be leveraged to bring constituencies to the table who otherwise might resist participating in discussions of democratizing local culture. 


\subsection{Promoting Self-promotion: The Art No One Wants to Master}

One of the goals of our CAMPOS initiative (recall that CAMPOS stands for "Center for the Advancement of Multicultural Perspectives on Science") was to mentor affiliated faculty in the skills essential to succeeding in the academy. One of these skills is self-promotion. Meritocracies reward accomplishment; consequently, faculty must be able to articulate their accomplishments, especially in the context of collaborative work. So we held workshops for CAMPOS scholars (and more broadly for junior faculty, postdoctoral fellows, and graduate students) on how to make the case for accomplishment. In the process, we learned that the constant need in for selfpromotion-for securing competitive grants, for publishing in prestigious journals, and for invitations to present work at major conferences, all of which are necessary for career advancement-discouraged pursuing academic careers in STEM. Graduate students in particular cited this issue as a major factor for preferring industry over academia.

Workshop participants linked self-promotion to the negative trait of bragging, or boasting, regardless of who was doing it, and they expressed reluctance to carry on this tradition. Members of underrepresented groups relayed incidences where selfpromotion backfired: boasting was seen as "covering up" for contributions claimed but not actually made. "Credit" generally accrued to those with more power on the research team, and many had witnessed STEM researchers successfully use the work of others for their own advancement. Consistent with this, some faculty believed that, in having to self-promote, they were forced to underplay the role of junior colleagues, postdoctoral fellows, and students. Participants, not without warrant, viewed reliance on self-promotion as a barrier to fairness in the review process.

We were acutely aware that reluctance to self-promote is not independent of stereotypical gendered and cultural expectations (Kandiko Howson et al., 2018; Van der Lee \& Ellemers, 2018; Võ, 2012). For example, assertive behaviors, often rewarded in men, are less valued in women (Van der Lee \& Ellemers, 2018). We were therefore prepared to "school" particpants in the art of self-promotion so that women and URM faculty could "boast with the best of them." We underestimated the extent to which training in self-promotion would be perceived as reinforcing an unattractive feature of STEM in academia. Participants simply didn't want to play that game. We therefore shifted discussion to center on "accuracy in reporting of accomplishment" rather than "the art of self-promotion." This led to a broader discussion of how to write and interpret candidate's statements submitted in support of advancement.

\section{Understanding Local Culture}

The second major issue negatively affecting cultural change is failing to understand the nuances or idiosyncrasies of the existing local culture. Bringing in external groups 
to help facilitate change is likely to be unproductive if they do not understand this culture. It is imperative, therefore, that members of the organization to be changed lead that change. Because the immediate locus of change in our case was the STEM fields and not the university as a whole, leaders needed to be UC Davis STEM (and "STEM-adjacent") faculty. STEM scholars typically do not study the causes and consequences of social inequality either inside or outside the academy, so the first order of business was to learn about and understand the broad issues affecting inclusion. Our coalition of transformational "change leaders" spent the first two years of our program doing just this, enlisting the help of others along the way. Invited speakers sparked discussions of broad social issues. Group retreats enabled discussion and debate about how to best create a more-inclusive community within our own local culture. An external advisory board informed our actions, based on their collective experience and knowledge; its members also provided critical evaluative feedback on our ideas and progress. This type of learning for those spearheading transformational change is essential for generating a viable, tactical plan. Three main elements of the local culture are key to assess in this process: (1) its existing ("innate") commitment to diversity and inclusion, (2) the practical effectiveness of that commitment, and (3) processes currently in place to foster engagement in and accountability for that commitment across the community.

\subsection{Community Commitment to Diversity}

An organization's or institution's commitment to diversity is often reflected in the diversity of its membership and leadership. However, a group may appear diverse based on outward appearances, but in practice may not be inclusive if some members' contributions are not equally valued and respected. The expressed shared values of the community are also an important indicator of commitment to diversity and inclusion. For example, prior to the ADVANCE program, UC Davis had already developed a document on "Principles of Community," which was endorsed broadly by leaders across the institution. The principles were developed after extensive discussion and have been reaffirmed several times since their initial inception in 1990 (Office of Campus Community Relations, n.d.). These principles establish a "community identity" of shared values. They are repeated here:

\section{Principles of Community}

The University of California, Davis, is first and foremost an institution of learning, teaching, research and public service. UC Davis reflects and is committed to serving the needs of a global society comprising all people and a multiplicity of identities. The university expects that every member of our community acknowledge, value, and practice the following guiding principles.

We affirm the dignity inherent in all of us, and we strive to maintain a climate of equity and justice demonstrated by respect for one another. We acknowledge that our society carries within it historical and deep-rooted injustices and biases. Therefore, we endeavor to foster mutual understanding and respect among the many parts of our whole. 
We affirm the right of freedom of expression within our community. We affirm our commitment to non-violent exchange and the highest standards of conduct and decency toward all. Within this context we reject violence in all forms. We promote open expression of our individuality and our diversity within the bounds of courtesy, sensitivity and respect. We further recognize the right of every individual to think, speak, express and debate any idea limited only by university regulations governing time, place and manner.

We confront and reject all manifestations of discrimination, including those based on race, ethnicity, gender and gender expression, age, visible and non-visible disability, nationality, sexual orientation, citizenship status, veteran status, religious/non-religious, spiritual, or political beliefs, socio-economic class, status within or outside the university, or any of the other differences among people which have been excuses for misunderstanding, dissension, or hatred. We recognize and cherish the richness contributed to our lives by our diversity. We take pride in all our achievements, and we celebrate our differences.

We recognize that each of us has an obligation to the UC Davis community of which we have chosen to be a part. We will strive to build and maintain a culture and climate based on mutual respect and caring.

Although principles such as these lack the force of law, and although they can be interpreted as merely symbolic, they do establish assumptions, expected attitudes, and modes of behavior for the campus community. In short, they define the shared values of the collective culture. The Principles of Community were re-endorsed multiple times over the ensuing 30+ years. The ADVANCE program was rooted in that community identity.

\subsection{Assessing Commitment to Diversity}

It is not sufficient to simply state a commitment to diversity and expect everyone to act accordingly, especially given the fact that barriers to inclusion derive from society at large and manifest in local culture in different ways. It's important to assess whether and to what extent a commitment to diversity is widely embraced (as well as what "diversity" means to those queried). A common method is to compare the diversity of an organization to a control population. In our case, this would mean comparing the diversity of our faculty community to that of the undergraduate student population and then to compare that student population to the diversity of California itself. One can also examine the pool of Ph.D. awardees to determine if "pipeline" issues exist with respect to a loss of diversity over time as a career track progresses. Focusing solely on supposed pipeline issues, though, can detract from addressing bias and discrimination within the culture of the target community.

Surveys of job satisfaction among different groups within a community can help reveal potential problems. If respondents regardless of identity category find the work environment to be positive with regard to resources, opportunities, and standards for merit and promotion, this may indicate that the organization as a whole values diversity and inclusion. It is also important to assess retention issues, because the existence of bias can encourage targeted individuals to leave. 
Complaint processes are important as well, as they may help develop suitable interventions when critical issues are identified. But because bias is a learned behavior that starts early (see Quintana et al., 2006), and because early experiences will vary across communities (especially in departments with international faculty), negative sanctions or penalties for individuals can be counterproductive. A proactive approach is better, in which community values are shared with new members through open and transparent conversations.

\subsection{Accountability for Commitment to Diversity}

An organization may agree to "commit" to diversity, equity, and inclusion for a variety of reasons. Legal requirements and even financial incentives may foster such a commitment but when the external pressure to appear committed lessens, the "commitment" also lessens ("talking the talk but not walking the walk"). Thus, the entire community must assume accountability for creating and sustaining an inclusive workplace, irrespective of external pressures.

Genuine institutional transformation requires embedding the responsibility for inclusion in all tiers of an organization. The following questions are critical to ask: Is there a culture of "blaming the victim" in cases of harassment or microaggression? Are the targets of harassment or microaggression the only ones who speak out against it? Do they feel pressured to not complain? Is there training in how to deal with harassment and microaggression? Does the culture support open discussion of these and other discriminatory actions?

In hierarchical or tiered organizations, it is important that "peers of the tier" speak out against bias and discrimination. The stratified nature of academic institutions can make it difficult for individuals to report problems without fear of retribution against those with more power. Silence from supervisors or colleagues validates microaggressions and harassment. Therefore, reporting and resolution processes must account for hierarchy in addressing such issues. For example, it is extremely difficult for a faculty member to challenge the behavior of a department chair or dean. Schools and colleges, as well as departments, must develop guidelines for "safe" communication without risk of reprisal, bypassing existing lines of authority if necessary. In some instances, another faculty member may be needed to corroborate the problem, or be present when it is brought to the attention of others outside the department, college, or institution. A process that ensures open and transparent communication about bias and discrimination must be developed, vetted by faculty, and fully supported by the academic leadership.

Many organizations practice what they call "restorative justice" as an attempt to mediate negative exchanges surrounding microaggressions. Restorative justice is an approach in which victim and perpetrator are brought together to discuss who was harmed, how, and what the perpetrator can do to repair or mitigate the harm (Zehr, 2002/2014). Critics of this process point out that offenders' apologies to victims, even genuinely expressed, can imply that the victim is hypersensitive and 
the offender simply failed to recognize his or her hypersensitivity. Better processes involve acknowledgment of the offense against the shared values of the community. Microaggressions should be seen as harmful to the entire community, not simply an individual or single identity category; likewise, restitution should be made to the community as well as the individual. The best scenario is for all members of the community to be aware of the negative effects of stereotyping before any act of microaggression occurs in the first place, whether overtly or covertly.

As discussed in the Chapter, 'Barriers to Inclusion: Social Roots and Current Concerns', microaggressions occur, for example, when faculty of color are told they got their job based on their race or ethnicity, not because they met the standards of the search committee and their academic excellence was a match for the position and the department. Mexican Americans may be asked where they are "from," and when they state a geographic location in the United States, they are again asked, "but where are you really from?" Department faculty may naively, even proudly, assert that they are both "gender blind" and "color blind," which itself is a microaggression because it denies the ongoing existence of racial and gender oppression; choosing not to see it does not make the oppression disappear. Can we better sensitize faculty to microagressions so as to actively reduce or prevent them entirely (Forrest-Bank \& Jenson, 2015)?

Interventions to prevent microaggressions are clearly needed across a wide range of social institutions; in university settings, interventions must be adapted to meet the needs of faculty coming from various cultural and national backgrounds. Prevention programs should expose department faculty to (1) clear examples of microaggressions, (2) ways to recognize both common and unique forms of microaggressions in relation to gender, ethnicity, and race, and (3) sensitive communication strategies to use when microaggressions occur.

Relatedly, another important component of assessing existing culture is understanding the nature of complaint protocols, including where responsibility for resolution lies, who is ultimately accountable for remedying the harm, and the nature of community support for the victim. Equally important is correct assessment of the consequences for the offender. Often consequences focus on expecting the offender to override unconscious bias, despite evidence suggesting this is difficult if not impossible. Consequences that enable positive future social interaction with the targeted individual or group, rather than focusing on apologies alone, may be more effective in addressing the dehumanization associated with stereotyping and discrimination. The human brain has been described as a "hypothesis testing machine" (Hohwy, 2013) with respect to assumptions about identity. So, creating circumstances that enable the unconscious brain to "disprove" a social hypothesis are important. Providing the opportunity to discuss and learn that a given stereotype is incorrect may be more effective than attempts at behavioral suppression of the hypothesis that generated it.

Knowing the actual attitudes, values, and assumptions of faculty within a given local culture is a necessary prerequisite for meaningful institutional transformation. As discussed in the Chapter, 'Data-Driven Decision-Making', UC Davis was fortunate to have reliable tools such as the COACHE (Collaborative on Academic Careers in Higher Education) survey, which collected data on faculty perceptions of various 
work issues as well as workplace culture. Data can be broken down by gender, race, and other identity categories to identify patterns or trends. Such tools are important both in validating impressions of the local academic culture and in determining needed areas of intervention. One advantage we had in planning our ADVANCE initiatives was the high value faculty placed on collaborative research, as indicated by the COACHE surveys in both 2012-13 and 2016-17. UC Davis is a collaborative institution in part because of the existence of interdisciplinary "graduate groups" that span the more traditional ("siloed") department structure, enabling multidisciplinary research. Although disciplinary diversity is not the kind of diversity that the ADVANCE program targets, it can establish a foundation for respecting and valuing differing perspectives, approaches, and research contributions.

\section{Lessons Learned}

In the following section, we provide a summation of our own experiences in adapting expert advice to the idiosyncrasies of our local culture. Academic institutions envision themselves as meritocracies-participants' status in the hierarchy is earned rather than granted or inherited. Many undergraduates receive degrees in STEM and if they do well enough can be accepted to graduate school and obtain an advanced degree. Successful graduate students become postdoctoral fellows, successful postdoctoral fellows secure positions as junior faculty members, and faculty members then advance through the ranks to full professor and potentially administrative leadership. Each stage is associated with meritorious achievement and assessment of achievement is presumed to be objective. However, meritocracies often subtly (or not so subtly) create and sustain barriers to inclusion. If advancement is deemed a reflection of success, lack of advancement can mean failure (Mijs, 2014). Barriers to inclusion may be ignored or denied, such that lack of achievement becomes associated with lack of effort and/or innate talent rather than the existence of the barriers.

\subsection{Commitment to an Equitable Meritocracy}

Meritocracies are problematic because they are presumed to be objective, but "objectivity" itself is subjectively defined by those who most benefit from it. One consequence is that members of under-represented groups are "included" on terms set by others. Once they rise in career status within the system as is, they may affirm the existing relationship between merit and success and minimize the impact of barriers to inclusion, effectively suggesting "if I can make it, so can you"-a sentiment we heard many times during focus-group meetings with URM STEM faculty on campus. This can then reinforce the perception of majority-group members that the local culture is fine and doesn't need changing because minority-group members can excel if they want to. Related to this is the potential for a meritocracy to consider its 
own hierarchical structure as insulated from or unrelated to other social hierarchies: that white male faculty are overrepresented in the upper ranks of STEM in academia is presumed to reflect objective criteria for advancement (criteria that can absorb qualified others as they come up through the ranks), regardless of how racism and sexism might be operating in the "real" world.

Meritocracies-especially those that are seen as "objective"-pose significant barriers to creating an inclusive academy because the phenomena that form barriers to inclusion, such as unconscious bias, also shape assessments of merit (see also Stewart \& Valian, 2018). A more desirable goal is an equitable meritocracy, by which we mean the criteria for achievement are ecumenical, prestige is not used as proxy for achievement, the potential for implicit bias in evaluation and promotion is openly discussed, and mentoring and community engagement are valued/rewarded alongside or as part of research productivity. Securing commitment to an equitable meritocracy is imperative for institutional transformation, particularly when the local culture is, by contrast, firmly rooted in the ideals of an objective meritocracy that assumes a level playing field for all, regardless of social identity or background. In fact, early naysayers criticized the UC Davis ADVANCE program as unnecessary, since we had "diverse" faculty, thereby proving we were already inclusive. A key task of the ADVANCE program, then, was teaching faculty how diversity can exist without inclusion and how unconscious bias and other barriers to inclusion manifest within "objective" academic meritocracies. The Strength Through Equity and Diversity (STEAD) training, outlined in the Chapter, 'Assessing Institutionalized Bias', was indispensable in this regard, enabling both peer-to-peer learning and concrete policy change. Now required of all search committees involved in faculty recruitment, the STEAD training consistently gets high marks in faculty evaluations across fields and disciplines.

\subsection{Policy and Procedures for Advancement: Data-Driven and Transparent}

STEM fields in general and our local culture in particular are data-driven. Scientists are often skeptical that academic meritocracies are tainted by subjective bias, especially implicit bias. Although a large body of research documents systemic bias in the academy (reviewed in Stewart \& Valian, 2018), it is important to assess local institutional data because, as discussed in the Chapter, 'Data-Driven Decision-Making', the data must support the rationale for transformation. If the academic culture itself is data-driven, the strategic vision for inclusion must also be data-driven. Lack of diversity among faculty has many causes_lack of diversity in hiring pools, pipeline disparities, bias in hiring, bias in advancement, poor work climates leading to failures in retention, etc. Moreover, the specific issues that most affect faculty diversity can vary by discipline; there may not be one solution for all STEM fields. A deep dive into the data can help pinpoint the nature of a problem and focus efforts to fix 
it. This is not necessarily simple or straightforward. For example, equity analyses of overt measures such as time to promotion and salary can suggest that all is well and there is no documentable bias. Such was the case at our own institution: after multiple rounds of salary equity analysis and adjustment, some concluded there was no mandate for transformation and that lack of diversity must stem from "forces beyond our control" (competition for excellent candidates, inability to hire a candidate's partner, a non-diverse selection pool). However, climate survey and retention data did reveal patterns of exclusion and dissatisfaction consistent with implicit bias and other barriers to inclusion. This example underscores the importance of knowing which data to look at and what different types of data can (and can't) tell you. It is also important to be completely transparent with data even when specific findings do not support change.

Determining data-driven policy for establishing an equitable meritocracy is a key challenge; another is ensuring complete transparency of decision-making and criteria for advancement within the institution. Analysis of the COACHE survey results revealed high satisfaction with the clarity of criteria for advancement and their fairness in application. The 2012-13 COACHE survey did, however, identify a need for a more comprehensive system of mentoring, particularly for junior faculty. As a consequence, the UC Davis LAUNCH program, discussed in the Chapter, 'Mentorship, Sponsorship, and Professional Networking', was implemented and institutionalized. The higher "scores" given to mentoring in the 2016-17 survey point to the success of that program. Thus, survey tools can assess and provide data to guide action, as well as provide data that document the impact of changes actually made.

\subsection{Walking the Walk: Peer-Driven Learning and Community Engagement}

Creating a more-inclusive climate is a noble goal, though not one easily achieved. Since it is well-known in academia that senior administrators must obtain resources, faculty may view new initiatives with suspicion as to their true purpose. Is the institution actually committed to change or instead committed to garnering resources associated with a stated commitment to change? Given the nature of barriers to inclusion and the necessity of full community support for dismantling them, peer-driven community learning that vets a strategic vision through collaborative conversations across academic units is more effective than top-down, "mandated edification."

In seeking to avoid mandated edification in favor of community engagement, we were fortunate that the University of California was established as a system of shared governance, meaning the responsibility for running the institution is shared between administrators and faculty, at least ideally. Mechanisms exist for review of proposed policy changes by the governing body of the faculty-the Academic Senate. The very existence of this structure ensured established channels of communication to engage 
the broader faculty in developing a vision and plan for institutional transformation, and we took full advantage. The ensuing conversations enabled organizational learning-meaning, members of the organization learn how structures, practices, goals, and values (both explicit and implicit) shape the organization.

Research analyzing cultural change within organizations often cites the need to get "early wins" (Quinn \& Cameron, 1988), yet the very nature of barriers to inclusion makes early wins unlikely in an academic setting. This is another reason why CAMPOS was such a critical component of our ADVANCE Program: from the beginning, it made visible our commitment to inclusion, and helped us realize that commitment through a structured network/community of highly-accomplished interdisciplinary scholars.

New STEM faculty with a track record of commitment to diversity, equity, and inclusion are eligible for nomination as CAMPOS scholars. Although the role is not limited to junior faculty or to members of underrepresented groups, early-career Latina (and other women of color) faculty are core participants and their commitment to a more inclusive culture is, logically, partly rooted in their own experiences. Changing the culture of a meritocracy cannot be driven only or even primarily by those in the upper tiers of that meritocracy, since they are products of the status quo and may have a vested interest in maintaining it. Thus, CAMPOS scholars both embody institutional change and help drive it from "the bottom up." CAMPOS is discussed in more detail in the Chapter, 'Seeing Self: The CAMPOS Model'.

\section{Conclusion}

Successful institutional transformation requires understanding existing institutional culture, the nature of the change needed, and the best data-proven mechanisms to enable that change. A more inclusive culture-one that is welcoming to historically underrepresented groups - must not only recognize and understand the social, cultural, and historical factors enabling bias and discrimination, it must also recognize and confront the negative assumptions that some faculty associate with diversity and inclusion - primarily, that diversity and inclusion are antithetical to excellence. In challenging this assumption, it is important that faculty and administrators alike not point fingers or alienate colleagues whose buy-in is critical for moving forward. The focus must instead be on inclusivity of process. The vision for institutional transformation must be embraced and modeled by leadership but incorporate all levels of the institution. The vision must drive a strategic action plan - a plan animated not by incentives and resources but by a commitment to the value and benefits of knowledge production. 


\section{References}

CEO Action for Diversity and Inclusion. (n.d.). Retrieved from https://www.ceoaction.com

Davidson, C. N., \& Goldberg, D. T. (2010). The future of thinking: Learning institutions in a digital age. The MIT Press.

Forrest-Bank, S., \& Jenson, J. M. (2015). Differences in experiences of racial and ethnic microaggression among Asian, Latino/Hispanic, Black, and White young adults. The Journal of Sociology \& Social Welfare, 42, 141.

Furst-Holloway, S., Hardcastle, V., Douglas, H., \& Page, E. (2018). Sustaining advance programs: A correlational study. University of Cincinnati. Cincinnati: University of Cincinnati Leadership, Empowerment, and Advancement for Women STEM Faculty (LEAF).

Gray, J. A. (1990). Brain systems that mediate both emotion and cognition. Cognition and Emotion, 4(3), 269-288. https://doi.org/10.1080/02699939008410799

Hohwy, J. (2013). The predictive mind. Oxford University Press.

Kalev, A., Dobbin, F., \& Kelly, E. (2006). Best practices or best guesses? Assessing the efficacy of corporate affirmative action and diversity programs. American Sociological Review, 71(4), 589-617. https://doi.org/10.1177/000312240607100404

Kandiko Howson, C. B., Coate, K., \& de St Croix, T. (2018). Midcareer academic women and the prestige economy. Higher Education Research \& Development, 37(3), 533-548. https://doi.org/ 10.1080/07294360.2017.1411337

Kotter, J. P. (1995). Leading change: Why transformation efforts fail. Harvard Business Review, $59-67$.

Mijs, J. (2014). The unfulfillable promise of meritocracy: Three lessons and their implications for justice in education. Social Justice Research, 29, 14.

Office of Campus Community Relations. (n.d.). Retrieved from Principles of Community: https:// occr.ucdavis.edu/poc/

Quinn, R. E., \& Cameron, K. S. (1988). Paradox and transformation: Toward a theory of change in organization and management. Ballinger Publishing Company.

Quintana, S. M., Aboud, F. E., Chao, R. K., Contreras-Grau, J. M., Cross, W. E., Hudley, C. A., Hughes, D., Liben, L. S., Nelson-Le Gall, S., \& Vietze, D. L. (2006). Race, ethnicity, and culture in child development: Contemporary research and future directions. Child Development, 77, 1129.

Stewart, A. J., \& Valian, V. (2018). An inclusive academy: Achieving diversity and excellence. The MIT Press.

University of California, Davis Faculty Satisfaction Survey Reports (COACHE). (n.d.). Retrieved from University of California, Davis Faculty Satisfaction Survey Reports (COACHE): https://aca demicaffairs.ucdavis.edu/faculty-satisfaction-survey-reports-coache

Van der Lee, R., \& Ellemers, N. (2018) Perceptions of gender inequality in academia. In B. T. Rutjens \& M. J. Brandt (Eds.), Belief systems and the perception of reality (1st ed, pp. 63-78). Routledge. https://doi.org/10.4324/9781315114903

Võ, L. T. (2012) Navigating the academic terrain: The racial and gender politics of elusive belonging. In Gutiérrez y Muhs, G., Niemann, Y. F., González, C. G., \& Harris, A. P. (Eds), Presumed incompetent: The intersections of race and class for women in academia (pp. 93-109). University Press of Colorado.

Zehr, H. (2002/2014). The little book of restorative justice. Good Books. 
Open Access This chapter is licensed under the terms of the Creative Commons Attribution 4.0 International License (http://creativecommons.org/licenses/by/4.0/), which permits use, sharing, adaptation, distribution and reproduction in any medium or format, as long as you give appropriate credit to the original author(s) and the source, provide a link to the Creative Commons license and indicate if changes were made.

The images or other third party material in this chapter are included in the chapter's Creative Commons license, unless indicated otherwise in a credit line to the material. If material is not included in the chapter's Creative Commons license and your intended use is not permitted by statutory regulation or exceeds the permitted use, you will need to obtain permission directly from the copyright holder.

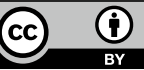

